\title{
Comparison of the Classical Method and SEE-FIM Protocol in Detecting Microscopic Lesions in Fallopian Tubes with Gynecological Lesions
}

\author{
Nermin Koc · Selçuk Ayas ${ }^{1}$ \\ Sevcan Arzu Arinkan ${ }^{1}$ \\ Departments of Pathology and ${ }^{1}$ Obstetrics and \\ Gynecology, Zeynep Kamil Maternity and \\ Pediatric Research and Training Hospital, \\ Istanbul, Turkey
}

\begin{abstract}
Background: The objective of this study was to compare the classical method and Sectioning and Extensively Examining the Fimbriated End Protocol (SEE-FIM) in detecting microscopic lesions in fallopian tubes with gynecological lesions. Methods: From a total of 1,118 cases, 582 with various parts of both fallopian tubes sampled in three-ring-shape sections and 536 sampled with the SEE-FIM protocol were included in this study. Pathological findings of cases with endometrial carcinoma, non-uterine pelvic malignant tumor, ovarian borderline tumors, premalignancy, and benign lesions were compared. Results: We detected two tubal infiltrative carcinomas among 40 uterine endometrioid adenocarcinomas, 15 serous tubal intraepithelial carcinomas in 39 non-uterine pelvic serous high-grade carcinoma cases, seven papillary tubal hyperplasias in 13 serous borderline tumor cases, and 11 endometriotic foci and four adenomatoid tumors among all cases sampled with the SEE-FIM protocol. Using the classical method, we detected only one serous tubal intraepithelial carcinoma in 113 non-uterine pelvic serous high-grade carcinoma cases and two papillary tubal hyperplasia cases in 31 serous borderline tumors. We did not identify additional findings in 185 uterine endometrioid carcinoma cases, and neither endometriotic focus nor adenomatoid tumor was shown in other lesions by the classical method. Conclusions: Benign, premalignant, and malignant lesions can possibly be missed using the classical method. The SEE-FIM protocol should be considered especially in cases of endometrial carcinoma, nonuterine pelvic serous cancers, or serous borderline ovarian tumors. For other lesions, at least a detailed examination of the fimbrial end should be undertaken.
\end{abstract}

Key Words: SEE-FIM method; Classical method; Fallopian tube lesions

\author{
Received: March 13, 2016 \\ Revised: June 11, 2016 \\ Accepted: June 16, 2016

\section{Corresponding Author} \\ Sevcan Arzu Arinkan, MD \\ Department of Obstetrics and Gynecology, \\ Zeynep Kamil Maternity and Pediatric Research and \\ Training Hospital, Istanbul 34660, Turkey \\ Tel: +90-505-683-7557 \\ E-mail: pataraa96@gmail.com
}

Clinical interest in the fallopian tube continues to increase. Recent studies on the carcinogenesis and origin of ovarian carcinoma have suggested tubal epithelium as a source of high-grade serous carcinoma (HGSC). ${ }^{1-4}$ Tubal carcinoma has been demonstrated in pathological specimens of BRCA1 and BRCA2 mutation carrier women who chose to have prophylactic salpingo-oophorectomy to reduce their risk of ovarian carcinoma. ${ }^{4}$ In addition to HGSC, low-grade serous carcinomas are thought to originate from the tubal epithelium, and papillary tubal hyperplasia (PTH) is considered a precursor to serous borderline tumors (SBT), noninvasive implants, and endosalpingiosis. ${ }^{4}$ In addition, a significant association of salpingoliths with SBT has been demonstrated. ${ }^{5}$

The fallopian tube has an indirect role in the pathogenesis of endometrioid and clear cell carcinomas of the endometrium and ovary. ${ }^{3}$ The presence of simultaneous or incidental lesions in fallopian tubes, the need for determination of their pathogenesis or their precursors, and the effects of fallopian tube metastasis on treatment modalities and on disease stage indicate the im- portance of fallopian tube sampling techniques. ${ }^{6}$

There are different approaches for sampling fallopian tubes. The pathology textbook Ackerman-Rosai Surgical Pathology recommends the classical sampling technique including collection of three "ring-shaped" sections from various parts of each tube. ${ }^{7}$ In Blaustein's Pathology of the Female Genital Tract, ${ }^{8}$ sampling of entire bilateral fallopian tubes with fimbrial ends is recommended for pelvic serous tumors and prophylactic salphingo-oophorectomies. However, for benign diseases and other malignant conditions, collection of at least one sample from each tube is recommended. ${ }^{8}$ The Association of Directors of Anatomic and Surgical Pathology recommends three sections for tubal carcinomas and at least three sections including isthmus, ampulla, and infundibulum/fimbria for routine cases. ${ }^{9}$

In this study, we aimed to compare the clasical method and Sectioning and Extensively Examining the Fimbriated End Protocol (SEE-FIM) in detecting microscopic lesions in fallopian tubes with gynecological lesions. 


\section{MATERIALS AND METHODS}

In the pathology department of our hospital, the SEE-FIM protocol has been used since 2012. Before that, fallopian tubes were sampled using the classical method involving collection of three "ring-shaped" sections from various parts of each tube. The SEE-FIM protocol includes amputation of each fimbria at the infundibulum, longitudinal sectioning of the fimbria, and extensive cross sectioning of the remaining tube at 2-mm intervals. ${ }^{10}$

This study was conducted on 1,118 patients who underwent total abdominal hysterectomy and bilateral salpingo-oophorectomy at our hospital from January 2006 to May 2014. The fallopian tubes were sampled by the classical method in 582 cases between 2006 and 2011, and 536 cases performed after 2011 underwent the SEE-FIM protocol. All sample slides were reexamined with light microscopy by two pathologists. Data on the macroscopic evaluations and other clinicopathological examinations were collected by chart review.

Cases were grouped according to the final diagnosis as endometrial carcinoma, non-uterine pelvic malignant tumors (ovarian, peritoneal, and tubal), ovarian borderline tumor and premalignant-benign lesions, and other tumors. Pathological findings of the classical and SEE-FIM protocols were compared between subgroups. Pelvic serous carcinomas (PSCs) were classified as "primary ovarian," "fallopian tube," and "primary peritoneal" according to Gynecologic Oncology Group criteria. ${ }^{11}$ In fallopian tube cancer cases, intact tubal parts were also examined.

Serous tubal intraepithelial carcinoma (STIC) was diagnosed as noninvasive tubal epithelium displaying marked nuclear atypia characterized by loss of polarity, increased nuclear/cytoplasmic ratios, increased nuclear size, hyperchromasia, irregular nuclear membranes, and chromatin distribution. In addition, absence of cilia and mitotic figures was also characterized as STICs. ${ }^{6} \mathrm{Im}$ munostainings for $\mathrm{p} 53$ and $\mathrm{Ki}-67$ were performed to diagnose STIC. ${ }^{12,13}$ p 53 signatures are defined as benign-appearing tubal epithelium with strong staining for $\mathrm{p} 53$ by immunohistochemistry and a low Ki-67 index. ${ }^{6}$ Immunohistochemistry was performed on formalin-fixed, paraffin-embedded tissue sections using a manual polymer detection system with citrate buffer heatinduced epitope retrieval. Pre-diluted ready-to-use primary antibodies were used including p53 (clone DO-7 + BP53-12v, Thermo Scientific, Waltham, MA, USA) and Ki-67 (clone SP6, Thermo Scientific).

Salpingoliths were described as mucosal and luminal calcifications that were frequently surrounded by bland epithelium in the fallopian tube. PTH was described as small rounded clus- ters of tubal epithelial cells and small papillae floating within the tubal lumen, with or without associated psammoma bodies, and demonstration of these findings with at least three papillae. The statistical difference between the two groups was examined by Fisher exact test.

The study was approved by the Institutional Review Board of Zeynep Kamil Women and Children Diseases Research and Training Hospital (IRB No. 143) and performed in accordance with the principles of the Declaration of Helsinki. Written informed consents were obtained.

\section{RESULTS}

This study included a total of 1,096 abdominal hysterectomy and salpingo-oophorectomy cases. Table 1 illustrates the number of cases in each group. Benign lesions, malignant neoplasms,

Table 1. Pathological diagnoses and number of cases in each group

\begin{tabular}{lcc}
\hline Pathology & $\begin{array}{c}\text { Classical method } \\
(n=582)\end{array}$ & $\begin{array}{c}\text { SEE-FIM protocol } \\
(n=536)\end{array}$ \\
\hline $\begin{array}{l}\text { Endometrial carcinoma } \\
\text { Non-uterine pelvic malignant tumor }\end{array}$ & 210 & 48 \\
$\quad$ Ovarian malignant tumors & 150 & 49 \\
Tubal malignant tumor & 11 & 9 \\
$\quad$ Peritoneal carcinoma & 5 & 3 \\
Ovarian borderline tumor & 44 & 17 \\
Premalignant and benign & 162 & 410 \\
lesions, other tumors & & \\
\hline
\end{tabular}

SEE-FIM, Sectioning and Extensively Examining the Fimbriated End Protocol. aPremalignant lesions (endometrial hyperplasia, cervical intraepithelial lesions), benign lesions (endometrial polyp, myoma), carcinomas of the cervix, vagina, and vulva.

Table 2. Clinical and pathological features of endometrioid adenocarcinoma cases

\begin{tabular}{lcc}
\hline Variable & $\begin{array}{c}\text { Classical method } \\
(\mathrm{n}=185)\end{array}$ & $\begin{array}{c}\text { SEE-FIM protocol } \\
(\mathrm{n}=40)\end{array}$ \\
\hline Age, mean (yr) & 60 & 61 \\
Tumor grade & 58 & 14 \\
1 & 70 & 18 \\
2 & 57 & 8 \\
$\quad 3$ & 34 & 8 \\
Myometrial invasion & 78 & 20 \\
$\quad$ None & 73 & 12 \\
$<1 / 2$ & 14 & 4 \\
$\quad>1 / 2$ & 9 & 5 \\
Lymph node metastasis & 2 & 0 \\
$\begin{array}{l}\text { Extrauterine extension } \\
\text { No. of cases with tubal } \\
\text { infiltrative carcinoma }\end{array}$ & & \\
\hline
\end{tabular}

SEE-FIM, Sectioning and Extensively Examining the Fimbriated End Protocol. $\mathrm{a} p=.031$ 
and premalignant lesions of the fallopian tubes in each group were evaluated in detail.

\section{Endometrial carcinoma}

Endometrioid adenocarcinoma was detected in 185 of 210 endometrial carcinomas using the classical method and was detected in 40 of 48 endometrioid malignant tumors using the SEEFIM protocol. Other cases were clear cell carcinoma, undifferentiated tumor, malignant mixed müllerian tumor, and serous carcinoma. The clinical and pathological characteristics of the endometrioid adenocarcinoma cases are shown in Table 2.

Among the endometrioid adenocarcinomas sampled by the

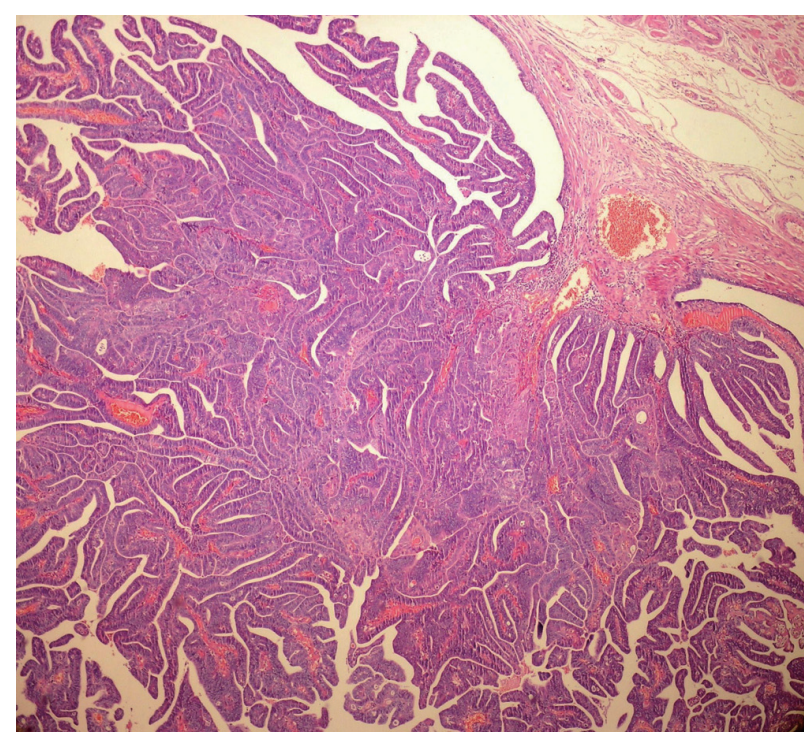

Fig. 1. Polypoid infiltrative endometrioid carcinoma extending to the tubal lumen.
SEE-FIM protocol, tubal infiltrative carcinoma was identified in two cases (Fig. 1). The clinical and pathological features of these cases are shown in Table 3. Polypoid lesions were detected in these two tubal infiltrative carcinoma cases, and these lesions showed similar microscopic features with lesions in the endometrium. Neither in situ nor invasive lesions were identified in fallopian tubes sampled by the classical method. This difference was statistically significant $(\mathrm{p}=.031)$. Tubal endometriotic foci were shown in four endometrioid carcinoma cases using the new technique, while two endometriotic foci were seen in fallopian tubes sampled by the classical method.

\section{Non-uterine pelvic malignant tumors}

Of non-uterine pelvic malignant tumor cases sampled by the new technique, 42 were serous carcinoma. Among these, there were eight tubal, three peritoneal, and 28 ovarian HGSCs. Serous carcinoma was detected in 113 of 166 non-uterine pelvic carcinoma cases sampled by the classical method. Among these cases, there were 85 ovarian, 11 tubal, and five peritoneal HGSCs (Table 4). Of all cases, p53 positivity was detected in 22 tubal epithelium samples. Among these, six tubal epithelium samples were identified as macroscopically benign with a low Ki-67 index. These samples were identified as "p53 signature" (Fig. 2). In cases sampled by the new technique, STIC was detected in 10 of 28 ovarian HGSCs and four tubal carcinomas. All lesions except one were located in fimbrial ends (93\%).

In peritoneal serous carcinomas, invasive serous carcinoma with a diameter of $0.2 \mathrm{~cm}$ was detected, and STIC was shown in the same case in the fimbrial end. STIC was identified in 15 of 39 HGSCs (40\%). Among the tubal carcinoma cases, STIC was

Table 3. Clinical and pathological characteristics of the tubal infiltrative carcinoma cases

\begin{tabular}{lccccccc}
\hline $\begin{array}{l}\text { Case } \\
\text { No. }\end{array}$ & $\begin{array}{c}\text { Age } \\
(\mathrm{yr})\end{array}$ & $\begin{array}{c}\text { Primary tumor } \\
\text { type }\end{array}$ & $\begin{array}{c}\text { Myometrial } \\
\text { involvement }\end{array}$ & $\begin{array}{c}\text { Primary tumor } \\
\text { grade }\end{array}$ & $\begin{array}{c}\text { Localization of tubal } \\
\text { involvement }\end{array}$ & $\begin{array}{c}\text { Size of tubal } \\
\text { involvement (cm) }\end{array}$ & $\begin{array}{c}\text { Other metastatic } \\
\text { sites }\end{array}$ \\
\hline 1 & 38 & Endometrioid & $<1 / 2$ & 1 & Fimbrial & 0.2 & Ovary, cervix \\
2 & 56 & Endometrioid & $>1 / 2$ & 2 & Ampullary & 0.3 & None \\
\hline
\end{tabular}

Table 4. Non-uterine pelvic carsinoma cases and STIC ratios identified by the classical method and SEE-FIM method

\begin{tabular}{lcccc}
\hline Histopathology & Classical method & STIC cases & SEE-FIM & STIC cases \\
\hline Ovary high-grade serous carcinoma & 85 & 0 & 28 & 3 \\
Ovary low-grade serous carcinoma & 12 & 0 & 10 & 0 \\
Ovary nonserous carcinoma $^{a}$ & 53 & 0 & 8 & 0 \\
Tubal serous carcinoma $_{\text {Tubal nonserous carcinoma }}^{\text {b }}$ & 11 & $1(9)$ & 1 & $4(50)$ \\
Peritoneum $_{\text {Total No. of cases }}^{c}$ & 0 & 0 & 3 & 0 \\
\hline
\end{tabular}

Values are presented as number (\%).

STIC, serous tubal intraepithelial carcinoma; SEE-FIM, Sectioning and Extensively Examining the Fimbriated End Protocol.

${ }^{a}$ Endometrioid, clear cell, mucinous, Krukenberg, malignant mixed mullerian tumor, granulosa; ${ }^{b}$ Endometrioid; ${ }^{c} p<.001$. 

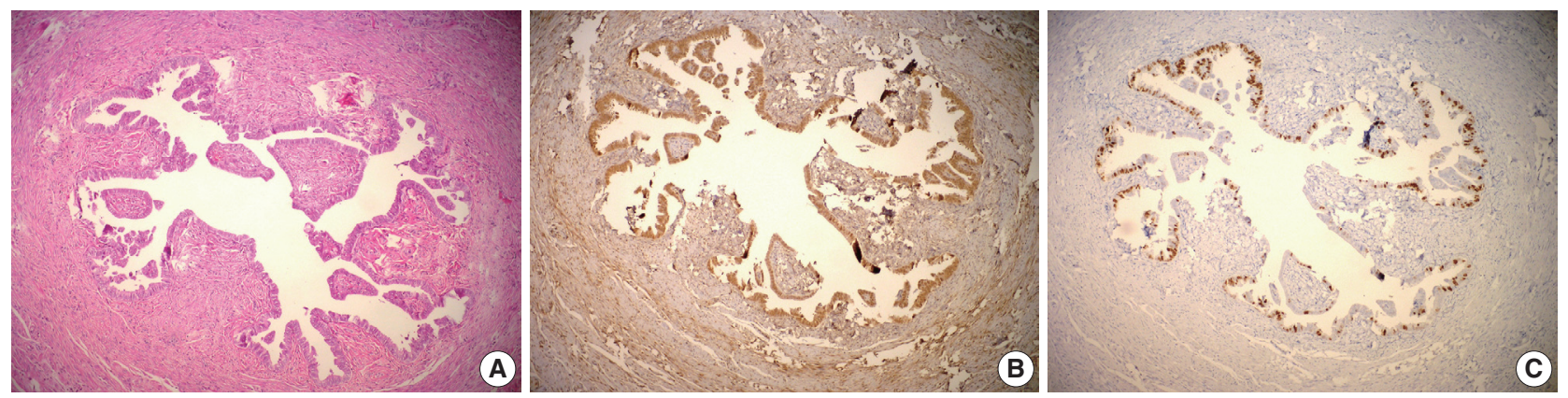

Fig. 2. Serous tubal intraepithelial carcinoma (A), positive immunostaining for p53 (B), and for Ki-67 (C).

Table 5. Clinical and pathological features of serous borderline tumors

\begin{tabular}{lcc}
\hline Variable & $\begin{array}{c}\text { Classical method } \\
(\mathrm{n}=31)\end{array}$ & $\begin{array}{c}\text { SEE-FIM protocol } \\
(\mathrm{n}=13)\end{array}$ \\
\hline Age, mean $(\mathrm{yr})$ & 44 & 40 \\
Bilateral & 24 & 9 \\
Microinvasion & 8 & 5 \\
Implant $^{\text {a }}$ & 3 & 2 \\
Endosalpingiosis $_{\text {No. of cases with papillary }}$ & 2 & 1 \\
tubal hyperplasia & 2 & 7 \\
\hline
\end{tabular}

SEE-FIM, Sectioning and Extensively Examining the Fimbriated End Protocol. almplants are noninvasive and nondesmoplastic.

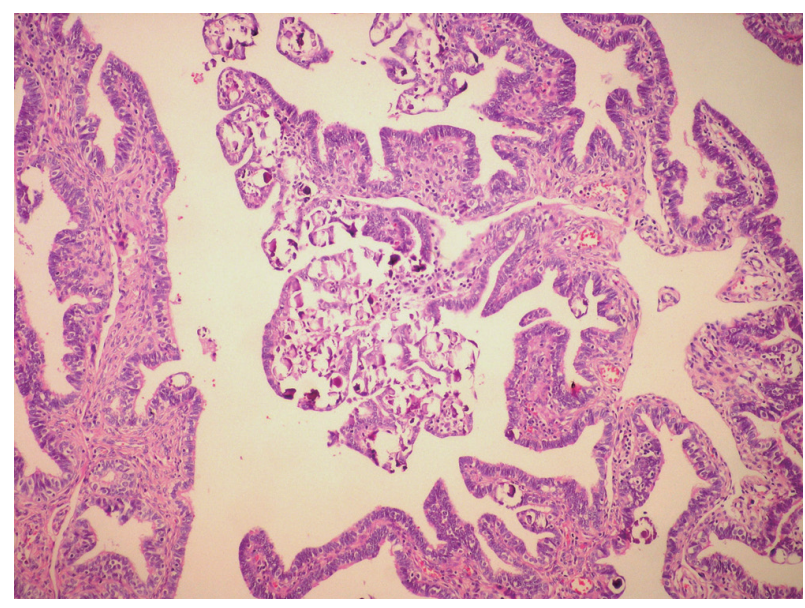

Fig. 3. Papillary tubal hyperplasia. Small rounded clusters of tubal epithelial cells and small papillae associated with psammoma bodies.

shown in only one case $(0.5 \%)$ by the classical method. This difference was statistically significant $(\mathrm{p}=.001)$. We did not detect any p 53 signature with the classical method, but six new cases were detected with the new method.

\section{Ovarian borderline tumors}

While serous borderline tumor was identified in 31 of 44 ovarian borderline tumors using the classical method, it was detect- ed in 13 of 17 cases in the SEE-FIM group. Other cases were mucinous, seromucinous, and endometrioid. The clinical and pathological features of the SBT in each group are shown in Table 5.

PTH was shown in seven of 13 cases (55\%) sampled by the SEE-FIM protocol (Fig. 3). One case was bilateral, four were diffuse, and four were focal lesions. Three of the focal lesions were located in the ampulla and infundibulum. PTH was detected in two of the cases with implants. In the classical method group, PTH was shown in two cases $(6 \%)$. There was a statistically significant difference $(\mathrm{p}=.001)$. Moreover, although salpingoliths were detected in two cases sampled by the new tecnique, it was not identified in the classical method group. Except for serous borderline tumor cases, PTH was not detected by either the classical method or the SEE-FIM protocol.

\section{Premalignant and benign lesions and other tumors}

Of 410 cases sampled by the new technique, tubal endometriosis and adenomatoid tumor were detected in seven and four cases, respectively. While five endometriotic foci were located in the infundibulum, two were in the ampulla. Adenomatoid tumors were located in the ampulla and infundibulum, with a mean diameter of $1.2 \mathrm{~cm}$ both at the serosa and subserosa. Neither tubal endometriotic focus nor adenomatoid tumor was identified in any of the 162 cases sampled by the classical method. There was a statistically significant difference between the two techniques regarding the diagnosis of adenomatoid tumor and endometriotic focus $(\mathrm{p}=.039)$.

\section{DISCUSSION}

\section{Endometrial carcinomas}

Detection of tubal lesions synchronous with endometrial cancer is important in management. Appropriate sampling of the tubes, ovaries, and lymph nodes is crucial in staging and treatment. The correct prognosis estimation is related to detection of tubal 
lesions in endometrial cancer. In our study, we detected two new tubal infiltrative carcinomas that were not seen by the classical method. As a result, the stage of one of these two cases was changed after the detection of the lesion. Since the other case demonstrated metastasis, detection of the lesion did not change the stage. Culton et al. ${ }^{14}$ reported synchronous endometrial and fallopian tube tumors in 13 cases. The sizes of the tumors ranged from $0.2 \mathrm{~cm}$ to $17.5 \mathrm{~cm}^{15}$ Kulac and Usubutun ${ }^{15}$ compared 100 fallopian tubes sampled by the classical method with 100 fallopian tubes with fimbrial end sampling and reported two invasive and two proliferative lesions that were not seen macroscopically. In our study, the sizes of the tubal lesions were 0.2 $\mathrm{cm}$ and $0.3 \mathrm{~cm}$, and they were not detected macroscopically. Culton et al. ${ }^{14}$ reported seven of 13 lesions using fimbrial end sampling, and Kulac and Usubutun ${ }^{15}$ identified three of four lesions using fimbrial end sampling. In our study, one of the two lesions was in the fimbrial end. Since tubal lesions can originate from lesions in the endometrium or endometrioid epithelium transformed from the tubal epithelium, studies on tubal lesions are important for determination of origin and pathogenesis of these tumors. Kulac et al. ${ }^{15}$ reported an association of endometriotic foci with tubal lesions in two of four cases. We did not identify any endometriotic focus in our cases.

\section{Non-uterine pelvic malignant tumors}

The majority of the non-uterine pelvic carcinomas are serous carcinomas that originate from the ovaries, fallopian tubes, or peritoneum. As non-uterine pelvic carcinomas have poor prognosis, the pathogenesis and origin should be well understood in order to develop new screening methods, new treatment modalities, and improved diagnosis at an early stage. STIC located in fimbria has been demonstrated as the origin of HGSC in recent studies. ${ }^{1-3}$ In addition to serous carcinoma, clear cell and endometrioid carcinomas have been thought to originate from endometriotic foci that are assumed to occur through retrograde menstruation. $^{1-3}$

In our study, we sampled the entire fallopian tubes, and STIC was shown in 15 of 39 cases with HGSCs. The rate was reported as 59\%, 52\%, and 20\% in studies by Przybycin et al., ${ }^{16}$ Kindelberg et al., ${ }^{17}$ and Tang et al., ${ }^{18}$ respectively. In our study, the percentages of ovarian, tubal, and peritoneal serous carcinomas in all non-uterine PSCs were changed from $72 \%, 20 \%$, and $8 \%$ to $45 \%, 50 \%$, and $5 \%$, respectively. Most lesions were located at the fimbrial end, and this finding is consistent with the other studies. No additional lesions in the fallopian tubes were detected in three endometrioid and one clear cell carcinoma cases sampled by the new technique. In non-uterine serous pelvic carcinomas sampled by the conventional method, STIC was identified in one case with HGSC. There were no additional lesions in the tubes in the endometrioid or clear cell carcinoma cases.

\section{Ovarian borderline tumors}

Regarding the origin of SBT, Kurman et $a l^{4}{ }^{4}$ reported that all ovarian and extraovarian low-grade serous proliferations originate from spilling and implantation of tubal epithelium in the form of PTH generated due to chronic inflammation. In their study, 20 of 22 cases (91\%) with noninvasive and invasive implants were associated with PTH. ${ }^{4}$ Similarly, Robey and Silva ${ }^{19}$ reported that $68 \%$ of SBT cases were associated with PTH.

Kurman et al. ${ }^{4}$ reported that PTH is mostly located in the ampulla; while the majority of lesions show a diffuse pattern, they can also be focal. Our study showed a lower percentage (55\%) of cases demonstrating an association of PTH with SBT sampled by the new technique. This difference may be due to the smaller number of cases with an implant in our study. The majority of focal lesions were located in the ampulla and infundibulum.

Yanai-Inbar et $a .^{20}$ reported that there was no statistically significant difference in detection of tubal pathology between sampling tubes from one section, two sections, or sampling the entire tube. Yanai-Inbar et al. ${ }^{20}$ analyzed the fallopian tubes of 48 SBT cases and found no difference between the study and control groups. In our study, we detected an association between PTH and ovarian borderline tumors in $6 \%$ of the cases sampled by the classical method. While diffuse lesions and random proliferations specific to this section were detected by the classical method, all PTH lesions were detected by the new technique.

Salpingoliths can be found in normal fallopian tubes. Kurman $e t a l^{4}$ and Seidman et al. ${ }^{5}$ have pointed out the association of salpingoliths with SBT. In our study, salpingoliths were found in $10 \%$ of SBT cases sampled by the new technique and were not demonstrated in SBT cases sampled by the classical method.

\section{Premalignant and benign cases and other tumors}

The pathogenesis of endometriosis and its association with malignancies remain interesting topics of gynecopathology. ${ }^{21,22}$ Endometrial tissue can be physiologically seen in the isthmus, but there is not enough data on the involvement of other areas.

In 410 fallopian tubes sampled by the new technique, we identified seven endometriotic foci $(2 \%)$. However, it was not shown in any of the fallopian tubes sampled by the classical method. Adenomatoid tumors are the most common benign 
neoplasm of the fallopian tubes. Their neoplastic potential and the fact that they can be misdiagnosed as other malignant or benign neoplasms should be considered during the management of these tumors. ${ }^{23}$ In our study, although we did not detect adenomatoid tumor by the classical method, four adenomatoid tumors were identified by the new technique.

It is possible to misdiagnose benign lesions, premalignant lesions, and malignant lesions using the classical method in pathological examination of the fallopian tubes. For this reason, the SEE-FIM protocol should be considered in cases of endometrial cancers, non-uterine pelvic serous cancers, or serous borderline ovarian tumors. The SEE-FIM protocol seems to have advantages for sampling of the entire fallopian tube. However, it may increase the surgical workload if it is used for all routine salpingectomy specimens. For cases with other benign, premalignant, and malignant lesions, at least a detailed examination of the fimbrial end of the fallopian tubes should be undertaken.

\section{Conflicts of Interest}

No potential conflict of interest relevant to this article was reported.

\section{REFERENCES}

1. Carcangiu ML, Peissel B, Pasini B, Spatti G, Radice P, Manoukian S. Incidental carcinomas in prophylactic specimens in BRCA1 and $B R C A 2$ germ-line mutation carriers, with emphasis on fallopian tube lesions: report of 6 cases and review of the literature. Am J Surg Pathol 2006; 30: 1222-30.

2. Yates MS, Meyer LA, Deavers MT, et al. Microscopic and earlystage ovarian cancers in BRCA1/2 mutation carriers: building a model for early BRCA-associated tumorigenesis. Cancer Prev Res (Phila) 2011; 4: 463-70.

3. Kurman RJ, Shih IM. Molecular pathogenesis and extraovarian origin of epithelial ovarian cancer: shifting the paradigm. Hum Pathol 2011; 42: 918-31.

4. Kurman RJ, Vang R, Junge J, Hannibal CG, Kjaer SK, Shih IM. Papillary tubal hyperplasia: the putative precursor of ovarian atypical proliferative (borderline) serous tumors, noninvasive implants, and endosalpingiosis. Am J Surg Pathol 2011; 35: 1605-14.

5. Seidman JD, Sherman ME, Bell KA, Katabuchi H, O'Leary TJ, Kurman RJ. Salpingitis, salpingoliths, and serous tumors of the ovaries: is there a connection? Int J Gynecol Pathol 2002; 21: 101-7.

6. Mehrad M, Ning G, Chen EY, Mehra KK, Crum CP. A pathologist's road map to benign, precancerous, and malignant intraepithelial proliferations in the fallopian tube. Adv Anat Pathol 2010; 17: 293302.

7. Rosai J. Rosai and Ackerman's surgical pathology. St. Louis: Mosby Elsevier, 2011.

8. Kurman RJ, Hedrick Ellenson L, Ronnett BM. Blaustein's pathology of the female genital tract. New York: Springer-Verlag, 2011.

9. Longacre TA, Oliva E, Soslow RA; Association of Directors of Anatomic and Surgical Pathology. Recommendations for the reporting of fallopian tube neoplasms. Hum Pathol 2007; 38: 1160-3.

10. Medeiros F, Muto MG, Lee $Y$, et al. The tubal fimbria is a preferred site for early adenocarcinoma in women with familial ovarian cancer syndrome. Am J Surg Pathol 2006; 30: 230-6.

11. Bloss JD, Liao SY, Buller RE, et al. Extraovarian peritoneal serous papillary carcinoma: a case-control retrospective comparison to papillary adenocarcinoma of the ovary. Gynecol Oncol 1993; 50: 347-51.

12. Kuhn E, Kurman RJ, Sehdev AS, Shih IM. Ki-67 labeling index as an adjunct in the diagnosis of serous tubal intraepithelial carcinoma. Int J Gynecol Pathol 2012; 31: 416-22.

13. Yemelyanova A, Vang R, Kshirsagar M, et al. Immunohistochemical staining patterns of p53 can serve as a surrogate marker for TP53 mutations in ovarian carcinoma: an immunohistochemical and nucleotide sequencing analysis. Mod Pathol 2011; 24: 1248-53.

14. Culton LK, Deavers MT, Silva EG, Liu J, Malpica A. Endometrioid carcinoma simultaneously involving the uterus and the fallopian tube: a clinicopathologic study of 13 cases. Am J Surg Pathol 2006; 30: 844-9.

15. Kulac I, Usubutun A. Microscopic lesions of fallopian tubes in endometrioid carcinoma of the endometrium: How effective are the macroscopic tubal sampling techniques? J Gynecol Oncol 2013; 24: 114-9.

16. Przybycin CG, Kurman RJ, Ronnett BM, Shih IM, Vang R. Are all pelvic (nonuterine) serous carcinomas of tubal origin? Am J Surg Pathol 2010; 34: 1407-16.

17. Kindelberger DW, Lee Y, Miron A, et al. Intraepithelial carcinoma of the fimbria and pelvic serous carcinoma: evidence for a causal relationship. Am J Surg Pathol 2007; 31: 161-9.

18. Tang S, Onuma K, Deb P, et al. Frequency of serous tubal intraepithelial carcinoma in various gynecologic malignancies: a study of 300 consecutive cases. Int J Gynecol Pathol 2012; 31: 103-10.

19. Robey SS, Silva EG. Epithelial hyperplasia of the fallopian tube: its association with serous borderline tumors of the ovary. Int J Gynecol Pathol 1989; 8: 214-20.

20. Yanai-Inbar I, Siriaunkgul S, Silverberg SG. Mucosal epithelial proliferation of the fallopian tube: a particular association with ovarian serous tumor of low malignant potential? Int J Gynecol Pathol 
1995; 14: 107-13.

21. Clement PB. The pathology of endometriosis: a survey of the many faces of a common disease emphasizing diagnostic pitfalls and unusual and newly appreciated aspects. Adv Anat Pathol 2007; 14: 241-60.
22. Modesitt SC, Tortolero-Luna G, Robinson JB, Gershenson DM, Wolf JK. Ovarian and extraovarian endometriosis-associated cancer. Obstet Gynecol 2002; 100: 788-95.

23. Quigley JC, Hart WR. Adenomatoid tumors of the uterus. Am J Clin Pathol 1981; 76: 627-35. 